\title{
Exploring the significance of the fluidized flow regime for avalanche hazard mapping
}

\author{
Dieter ISSLER, Peter GAUER
}

\begin{abstract}
Division of Natural Hazards, Norwegian Geotechnical Institute and International Centre for Geohazards, PO Box 3930, Ullevål Stadion, NO-0806 Oslo, Norway

E-mail: di@ngi.no
\end{abstract}

\begin{abstract}
Observational and experimental evidence suggests that it is important to explicitly account for the fluidized flow regime in avalanche hazard mapping due to its high mobility, intermediate density and high velocity. We explore the differences from conventional runout modelling by implementing an extension of the Norem-Irgens-Schieldrop (NIS) rheology in a simple mass-point model. When the dispersive stresses and the excess pore pressure equal the overburden pressure, the flow height increases and the density diminishes until a new equilibrium is reached, determined by the different density dependencies of the two parameters of the dispersive stresses. Fluidization requires sufficiently steep terrain; when it occurs it leads to substantially higher velocities than compared to the dense-flow regime. The model parameters are strongly constrained by their physical meaning and vary little between widely different avalanches. However, in all test cases we obtained better agreement between simulated and observed runout distances and pressure effects than with conventional models.
\end{abstract}

\section{INTRODUCTION}

For several decades, experiments and observations have indicated that dry snow avalanches realize all the main flow regimes discovered in non-cohesive granular materials, namely: the quasi-static (or frictional) regime in the denseflow part; the collisional and grain-inertia regimes in the partially or completely fluidized part in front of (and presumably also on top of) the dense core; and the macroviscous regime in the highly turbulent suspension flow (powder snow cloud). We found the fluidized part capable of breaking out of deep gullies, running up very high on counter slopes, covering large areas with substantial and often very hard deposits (Fig. 1) and of transporting huge snow clods that are then found scattered on the surface of the deposit (Issler and others, 1996) (Fig. 2).

The large avalanches that were artificially released at the Swiss test site Vallée de la Sionne in February 1999 provided us with live experience of the relevance of fluidized flow for hazard mapping. The radar shelter $50 \mathrm{~m}$ above the valley floor on the opposite slope was partially or completely covered by three avalanches with front speeds of 30$50 \mathrm{~m} \mathrm{~s}^{-1}$ and mean impact pressures between 10 and $70 \mathrm{kPa}$. This indicates densities of $10-50 \mathrm{~kg} \mathrm{~m}^{-3}$, in agreement with the visual observations of a semi-dilute front with large snow clods approaching at high speed and with the analysis of high-frequency pressure measurements (Schaer and Issler, 2001). In all three cases, the dense flow was deflected along the main valley axis as the clearly delineated deposits showed.

One may therefore expect that models that are capable of simulating the transition to and from the fluidized regime will make significantly different predictions from standard models. Our objective in this paper is to explore the potential implications of this new concept through a simple, preliminary mass-point model that simulates flow-regime transitions by the same mechanism we envisage for a future, more complete model. We briefly describe the model and its implementation and then discuss the results of applying it to several representative and quite different cases on which detailed information is available.

\section{A SIMPLIFIED MODEL FOR FLOW-REGIME CHANGES}

The collisional and grain-inertia regimes in granular flows have been studied much less than the frictional regime, which is dominant under typical laboratory conditions and admits stationary flows within a certain parameter range. The transition to a more dilute flow regime was probably observed by many researchers in the form of an accelerating flow when the limit slope for stationary flows was exceeded. Barbolini and others (2005) found a rapid, dilute head with a curved velocity profile, as expected in collision-dominated flows, in some of their chute experiments with plastic beads. Indications of diluted flow are also seen in video footage from experiments with ping-pong balls (Nishimura and others, 1998).

It is generally agreed that the coefficient of restitution of snow particles is rather low, presumably in the range $0.1-$ 0.4 . Velocity fluctuations generated by the bed roughness should therefore only be felt over distances of a few particle diameters (Rajchenbach, 2003) or on the scale of the roughnesses, which obviously impedes fluidization of the entire flowing layer. However, since fluidization is observed in nature and particle collisions must play an important role in it, we will base our model on dispersive stresses and allow for excess pore pressure to facilitate the flow-regime transition. Indeed, dynamic pressure of the order $(1 / 2) \rho_{\mathrm{a}} \dot{x}^{2}$ at the nose and suction of up to twice this magnitude on top of the front (Hampton, 1972) may offset up to one-third of the bed normal stress in medium to large avalanches, which is typically of the order of $2-5 \mathrm{kPa}$.

In the present exploratory phase, we content ourselves with a simple mass-point model enhanced by an additional dynamical property that may be taken either as the density $\rho$ or the flow height $h$. We include entrainment in the equation 


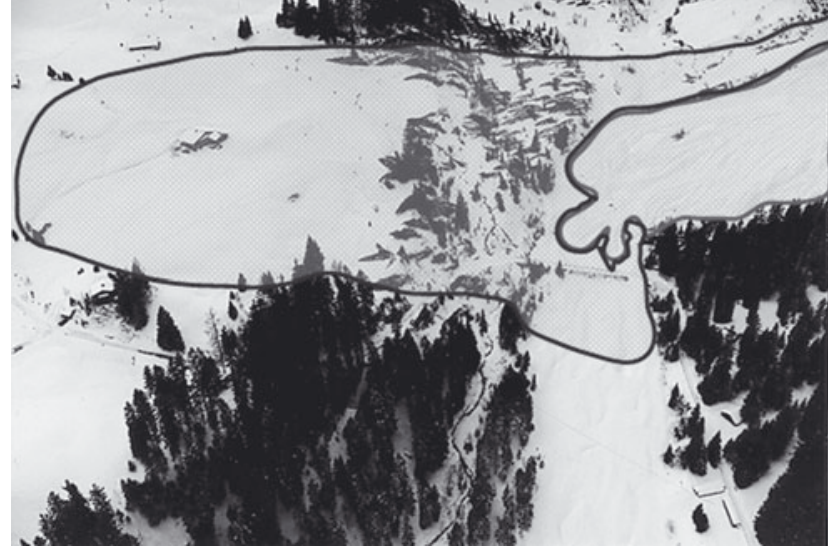

Fig. 1. Deposit area of the Albristhorn avalanche of 30 January 1995, Adelboden, Switzerland. The deposit of the dense part is clearly visible (greyish outline) and the area reached by the fluidized part is outlined in black. The suspension layer continued for several hundred metres uphill to the left (photograph courtesy of S. Keller).

of motion, but suppose that the additional mass lengthens the flow while the mass per unit footprint area $m_{0}$ remains constant. Along a slope parameterized by the coordinate $x$, with local slope angle $\theta(x)$, the equation of motion is therefore

$$
\ddot{x}=\mathrm{g} \sin \theta-\tau_{\mathrm{b}} / m_{0}-\dot{x} q_{\mathrm{e}} / m_{0},
$$

where $g$ is the gravitational acceleration, $\tau_{\mathrm{b}}$ is the bed shear stress and $q_{\mathrm{e}}$ is the mass entrainment rate, to be specified by the user.

Following the well-known Norem-Irgens-Schieldrop (NIS) model (Norem and others, 1987), we assume the slopenormal stress

$$
\sigma_{\mathrm{n}}=\rho h \mathrm{~g}_{\mathrm{n}}^{\prime}=\sigma^{(\mathrm{e})}+\sigma^{(\mathrm{d})}+p_{u}
$$

consists of the sum of the effective stress $\sigma^{(\mathrm{e})}$ transmitted by frictional interparticle contacts, the dispersive stress $\sigma^{(\mathrm{d})}$ in the slope-normal direction due to particle collisions and the excess pore pressure $p_{u} \cdot \mathrm{g}_{\mathrm{n}}^{\prime}=\mathrm{g} \cos \theta+\kappa \dot{x}^{2}$ is the slopenormal component of $g$, modified for centrifugal effects due to the path curvature $\kappa(x)$. (It is convenient to adopt the geotechnical convention which considers compressive stresses (pressures) positive.) We suppose that the effective stress gives rise to a frictional shear stress, $\tau_{\mathrm{C}}=\mu_{0} \sigma^{(\mathrm{e})}$, with a Coulombian friction coefficient $\mu_{0}$ that is constant as long as the intrinsic properties of the snow do not change.

The normal stresses tangential to the slope do not occur in our block model so that the dispersive contributions to the shear and slope-normal stresses in the NIS rheology reduce to

$$
\tau^{(\mathrm{d})}=\rho_{\mathrm{p}} \nu(\mathrm{c}) \dot{\gamma}^{2}, \quad \sigma^{(\mathrm{d})}=\rho_{\mathrm{p}} \nu^{\prime}(c) \dot{\gamma}^{2}
$$

in simple shear flow with shear rate $\dot{\gamma} \equiv \partial_{z} u_{x}$, if we neglect cohesion. Note that we scale these expressions by the intrinsic density of the particles $\rho_{\mathrm{p}}$ rather than the bulk density as in Norem and others (1987). We anticipate that the two viscosity parameters $\nu, \nu^{\prime}$ (with dimension $\mathrm{m}^{2}$ ) depend upon the volumetric particle concentration $c$, which relates to the bulk density through $\rho=c \rho_{\mathrm{p}}+(1-c) \rho_{\mathrm{a}} \sim c \rho_{\mathrm{p}}$, where $\rho_{\mathrm{a}}$ is the density of air. As in the one-dimensional (1-D) depthintegrated NIS model, we approximate the shear-rate profile

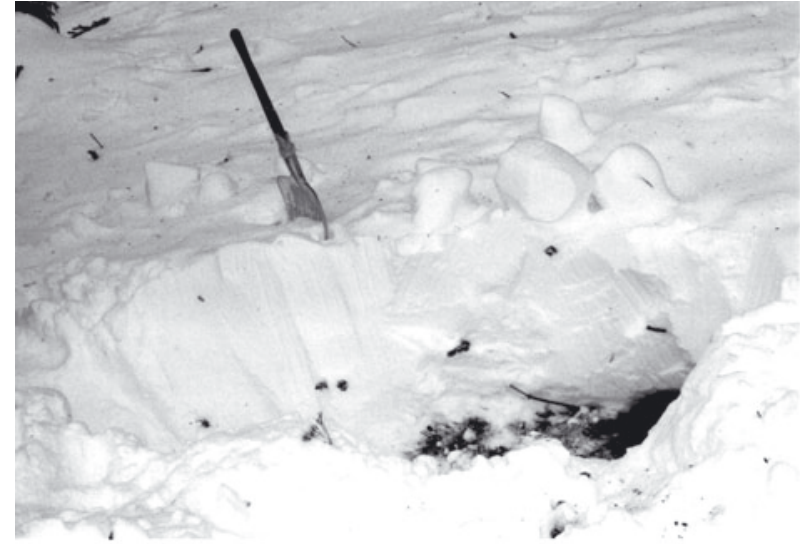

Fig. 2. Partial view of deposits of the fluidized part of the Schilteri avalanche of 11 January 1995, Seewis, eastern Switzerland. The fluidized part broke out of the 10-15 m deep narrow gully in a bend before eroding approximately $1 \mathrm{~m}$ of fresh snow and depositing $40-50 \mathrm{~cm}$ of compacted snow. Small snowballs were embedded in the deposit and large clods were scattered on the surface (photograph courtesy of M. Schaer, SLF).

by its equilibrium shape

$$
\dot{\gamma}(x, z, t)=\frac{5}{2} \frac{\bar{u}_{x}(x, t)}{h}\left(1-\frac{z}{h}\right)^{1 / 2},
$$

and identify the mean velocity $\bar{u}_{x}(x, t)$ with the centre-ofmass variable $\dot{x}(t)$. We thus obtain the dispersive contributions to the shear and normal stresses at the bed:

$$
\begin{aligned}
\tau_{\mathrm{b}}^{(\mathrm{d})} & =\frac{25}{4} \rho_{\mathrm{p}} \nu(c) \frac{\dot{x}^{2}}{h^{2}}, \\
\sigma_{\mathrm{b}}^{(\mathrm{d})} & =\frac{25}{4} \rho_{\mathrm{p}} \nu^{\prime}(c) \frac{\dot{x}^{2}}{h^{2}} .
\end{aligned}
$$

We simplify the spatially varying pore pressure field due to aerodynamic forces to a speed-dependent excess pore pressure at the bottom interface:

$$
p_{u}=\frac{1}{2} C_{\mathrm{L}} f_{C l}(c) \rho_{\mathrm{a}} \dot{x}^{2},
$$

where $\rho_{\mathrm{a}}$ is the air density, and the lift coefficient $C_{\mathrm{L}}$ is of the order 1 . The function $f_{C l}(C)$ parameterizes the loss of lift due to the permeability of the avalanche at low particle concentrations; our (arbitrary) choice is plotted in Figure 3.

Taking into account that the effective stress cannot become negative,

$$
\sigma^{(\mathrm{e})}=\max \left\{0, m_{0} g_{\mathrm{n}}^{\prime}-\left(\frac{25}{4} \rho_{\mathrm{p}} \frac{\nu^{\prime}}{h^{2}}+\frac{1}{2} C_{\mathrm{L}} f_{C l}(c) \rho_{\mathrm{a}}\right) \dot{x}^{2}\right\},
$$

we combine all these expressions to obtain the bed shear stress:

$$
\tau_{\mathrm{b}}=\mu_{0} \sigma^{(\mathrm{e})}+\frac{25}{4} \rho_{\mathrm{p}} \nu(c) \frac{\dot{x}^{2}}{h^{2}} .
$$

Fluidization occurs when $\sigma^{(\mathrm{e})}$ vanishes at

$$
\dot{x} \geq\left(\frac{\rho \mathrm{g}_{\mathrm{n}}^{\prime} h}{\frac{25}{4} \rho_{\mathrm{p}} \frac{\nu^{\prime}}{h^{2}}+\frac{1}{2} C_{\mathrm{L}} f_{C l} \rho_{\mathrm{a}}}\right)^{1 / 2} .
$$

In stationary flow down an inclined plane and with $p_{u}=0$, this condition is found to correspond to $\tan \theta \geq \nu / \nu^{\prime}$ (Norem and others, 1987), which is expected to lie in the range 0.61.0 according to shear tests on granular materials (Savage 
and Sayed, 1984). If the fluidization condition is fulfilled, the dispersive pressure drives the particles apart. As a consequence, collisions will become less frequent and both the normal and shear stresses will diminish until the overburden weight and $\sigma^{(\mathrm{d})}+p_{u}$ are in equilibrium again. A fundamental condition for an equilibrium to exist is that $\tau^{(\mathrm{d})}$ diminishes more slowly with decreasing density than $\sigma^{(\mathrm{d})}$ does.

There are few investigations of the density dependence of granular flow to guide our modelling, due to the intrinsic difficulty of such experiments. However, Savage and Sayed (1984) found, in a series of careful shearcell experiments probing different materials and volumetric concentrations in the range $0.44-0.54$, that (1) the stresses depend quadratically on the shear rate $\dot{\gamma}$ at high values of $\dot{\gamma}$ and lower particle concentration $c$ while (2) dry friction dominates at low $\dot{\gamma}$ and high $c_{\text {, }}(3)$ the effective friction $\tau / \sigma_{\mathrm{n}}$ in most cases increases moderately with $\dot{\gamma}$, but (4) increases quite significantly with decreasing $c$. Moreover, in the concentration range probed by the experiments, their results are well reproduced by the two-dimensional (2-D) numerical simulations of Campbell and Gong (1986). The latter showed how collisional transfer of momentum dominates at high $c$, and translational transfer (grain-inertia) at $c<0.2$. The analytic study of Pasquarell and others (1988) provides at least partial insight into the reasons why $\tau^{(\mathrm{d})}$ and $\sigma^{(\mathrm{d})}$ depend on the density in different ways. These studies clearly support the basic features of the NIS rheology.

As there are neither precise experiments with snow in the required parameter range nor completely realistic numerical simulations, we choose simple parameterizations for $\nu$ and $\nu^{\prime}$ that show the same qualitative behaviour as the mentioned experiments and simulations, at least for $c>0.05$ :

$$
\begin{gathered}
\nu^{\prime}(c)=A c^{-p}\left(c_{*}-c\right)^{-q}, \\
\nu(c)=k \nu^{\prime}(c)\left(1+b c^{-s}\right),
\end{gathered}
$$

where $c_{*}$ is the maximum concentration that allows shear. Figure 3 plots the functions $\nu^{\prime}(c), \nu(c)$ and their ratio for $C_{*}=0.6, A=10^{-4} \mathrm{~m}^{2}, b=1, k=0.2, p=0.5, q=1.5$ and $s=0.5$.

The simplified equation of motion in the slope-normal direction for $h(t)$,

$$
\ddot{h}(t)=2 \ddot{z}(t)=L_{1}(c) \frac{\rho_{\mathrm{p}}}{m_{0}} \dot{x}^{2}-L_{2}(c) \dot{h}-\mathrm{g}_{\mathrm{n}}^{\prime},
$$

allows us to determine $c$ from mass conservation $\left(c \sim m_{0} /\right.$ $h \rho_{\mathrm{p}}$ ) assuming that the density remains uniform throughout the depth and that the avalanche expands only in the $z$ direction. If the avalanche is not fluidized and the fluidization condition Equation (9) is not met, we set $\ddot{h}=0$ and $\dot{h}=0$. The coefficient $L_{1}(c)$ captures the effects of the dispersive and excess pore pressures:

$$
L_{1}(C)=\frac{25}{2} \frac{\nu^{\prime}}{h^{2}}+C_{\mathrm{L}} f_{C l}(C) \frac{\rho_{\mathrm{a}}}{\rho_{\mathrm{p}}} .
$$

The damping term describes the resistance of the air that has to enter or leave the flowing snow for the density to change. The corresponding coefficient $L_{2}(c)$ is obtained by applying Darcy's law, i.e.

$$
L_{2}(c) \sim \frac{k(c)}{\mu_{\mathrm{a}}} \frac{p_{\text {ref }}}{h^{2}(1-c)^{2}},
$$

where $\mu_{\mathrm{a}}$ is the dynamic viscosity of air, $p_{\text {ref }}$ is the reference

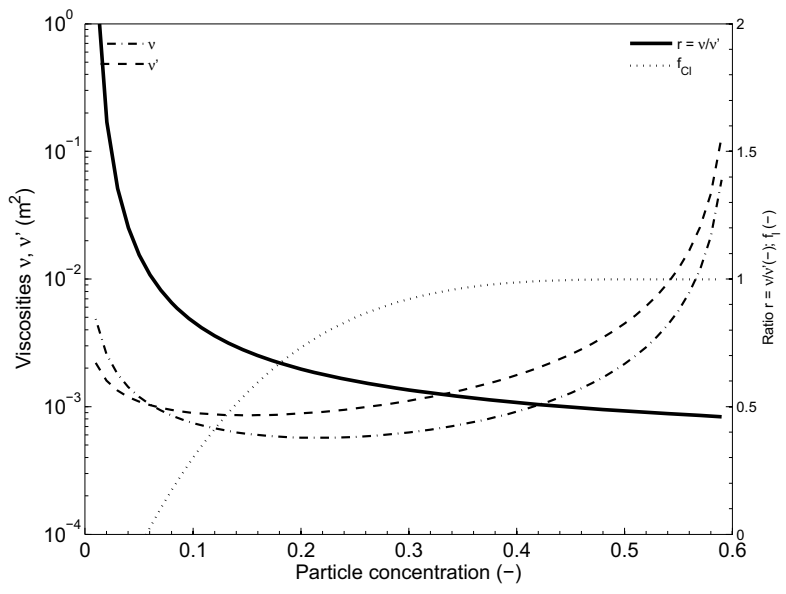

Fig. 3. Typical concentration dependence of the viscosities $\nu, \nu^{\prime}$ (Equations (10) and (11)) and their ratio. The permeability function $f_{C l}(c)$ introduced in Equation (6) is also plotted (scale on the right).

pressure (of the order of atmospheric pressure) and $k(c)$ is density-dependent permeability as recommended by Mellor (1977).

The equation of motion (Equation (1)) and the density evolution (Equation (12)) are solved with a fourth-order Runge-Kutta scheme. Input consists of the rheological parameters (including the particle concentration in the denseflow regime $c_{\mathrm{d}} \sim 0.45-0.55$ and the snow particle density $\rho_{\mathrm{p}} \sim 400-600 \mathrm{~kg} \mathrm{~m}^{-3}$ ), the initial conditions and the terrain profile. In the figures, it is termed B2E (block 2-equation model). The fluidization mechanism may be disabled for comparison purposes, leading to a block-model version of the NIS model (designated as NIS).

\section{TEST APPLICATIONS IN HAZARD MAPPING}

The main goals of our tests were to verify the proper functioning of the model, to determine the parameter ranges needed to reproduce observed fluidization behaviour, runout distances and speeds of widely different avalanches, and to assess the potential impact on avalanche hazard mapping. In a first step, we used avalanches from the experimental sites at Ryggfonn (RF) (http://www.leeds.ac.uk/satsie/ publications.html), Norway, and Vallée de la Sionne (VdlS), Switzerland, for which the initial conditions and velocity data are available. The density can be estimated from pressure measurements.

The VdIS 10 February 1999 avalanche (release volume 80000-100000 $\mathrm{m}^{3}$ ) developed a strongly fluidized head with density increasing from approximately $35 \mathrm{~kg} \mathrm{~m}^{-3}$ at the very front to $100-150 \mathrm{~kg} \mathrm{~m}^{-3}$ further back; it crossed the river and exerted pressures of about 15-20 kPa at the radar shelter. (The avalanche broke the entrance door to the shelter, located at the side and protected from direct impact. The pressure estimate was derived from the sudden pressure rise inside the bunker, which was experienced by one of the authors (D.I.).

Scattered snow clods more than $0.5 \mathrm{~m}$ in diameter were deposited beyond the shelter. Data on this avalanche have been analyzed and published by Schaer and Issler (2001), Vallet and others (2001) and Gauer and others (2007c). The RF 16 April 2005 avalanche, analyzed by Gauer and others $(2007 a, b)$, was much smaller, with a release volume of $10000-15000 \mathrm{~m}^{3}$. The fluidized front reached $40 \mathrm{~m} \mathrm{~s}^{-1}$, but 

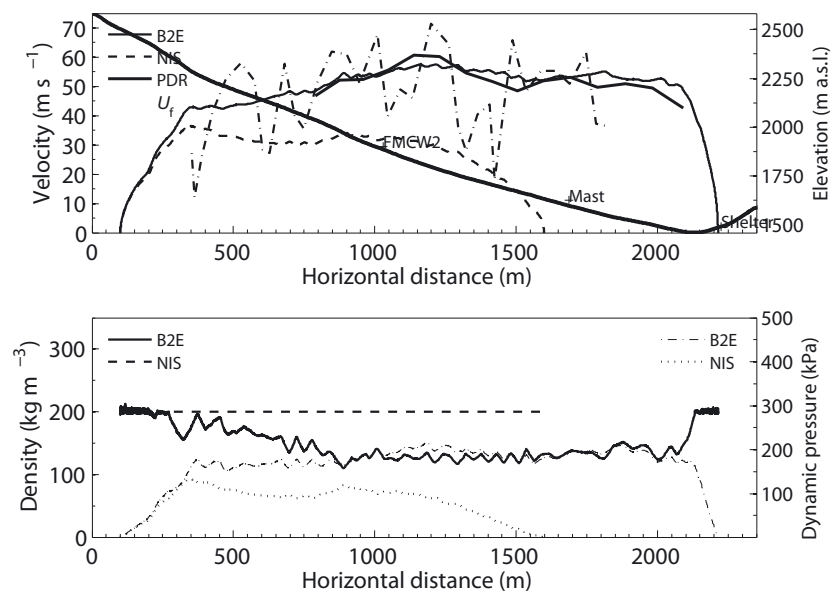

Fig. 4. Measurements and simulations of the 10 February 1999 avalanche event at Vallée de la Sionne. The velocities were measured by pulsed Doppler radar (PDR) (Gauer and others, 2007c), and the front velocity $\left(U_{\mathrm{f}}\right)$ was estimated from video footage. The same model parameters were used in both simulations, but fluidization was disabled in the simulation labelled ' $\mathrm{NIS}$ '.

the dense part was much slower due to entrainment of wet snow in the lower parts of the path. For both avalanches, the entrainment depth could be measured or estimated.

With similar model parameters in both cases, we obtained good agreement between measured and simulated velocities along the entire path (Figs 4 and 5). The RF 16 April 2005 avalanche, which ran into humid snow, required $\mu_{0}=0.575$ and $C_{\mathrm{L}}=1.3$. However, the VdIS 10 February 1999 event was best reproduced with lower dry friction, i.e. $\mu_{0}=0.5$, and more pronounced aerodynamic lift, i.e. $C_{\mathrm{L}}=1.7$. The mass-point version of the NIS model, run with the same parameter values, mimics the motion of the dense part and highlights the fact that fluidization leads to drastically higher velocities and longer runout distances. In order to highlight the difference between B2E and conventional two-parameter models, we created a variant of the often used PCM model (Perla and others, 1980). To this end we set $q_{e}=0$ and $\tau_{b}=\mu g \cos \theta+k \dot{x}|\dot{x}|$ in Equation (1); $\mu$ is the coefficient of dry friction and $k$ (with units of $\mathrm{m}^{-1}$ ) is the inverse of the parameter $M / D$ in the PCM model. In contrast to the original PCM model, we do not reduce the slope-parallel momentum by a factor of $\cos \Delta \theta$ where the slope abruptly changes by an angle $\Delta \theta$. In all models and test cases, it was found that the results are sensitive to entrainment and that extra friction due to centrifugal forces also has a non-negligible effect.

The medium-sized 18 January 2006 Rüchitobel avalanche in Davos, Switzerland (Issler and others, 2008), with an estimated release volume of $20000-40000 \mathrm{~m}^{3}$, showed substantial superelevation in a bend at $1750 \mathrm{ma}$ a.s.l. It produced flow marks characteristic of fluidized flow and strict confinement of the dense part to the gully bottom. The respective speeds were estimated as $28-38 \mathrm{~m} \mathrm{~s}^{-1}$ and $12-$ $18 \mathrm{~m} \mathrm{~s}^{-1}$. No tree damage was observed below $1700 \mathrm{~m}$ a.s.l., however. With $\mu_{0}=0.5$ and $C_{\mathrm{L}}=1.3$, the fluidized part was reasonably well reproduced, with the speed at the upper limit of the estimated range and with the pressures in the runout zone probably too high (Fig. 6). The latter shortcoming appears to be linked to the fact that the present model predicts relatively high fluidized densities around $150 \mathrm{~kg} \mathrm{~m}^{-3}$ with physically plausible parameter choices.
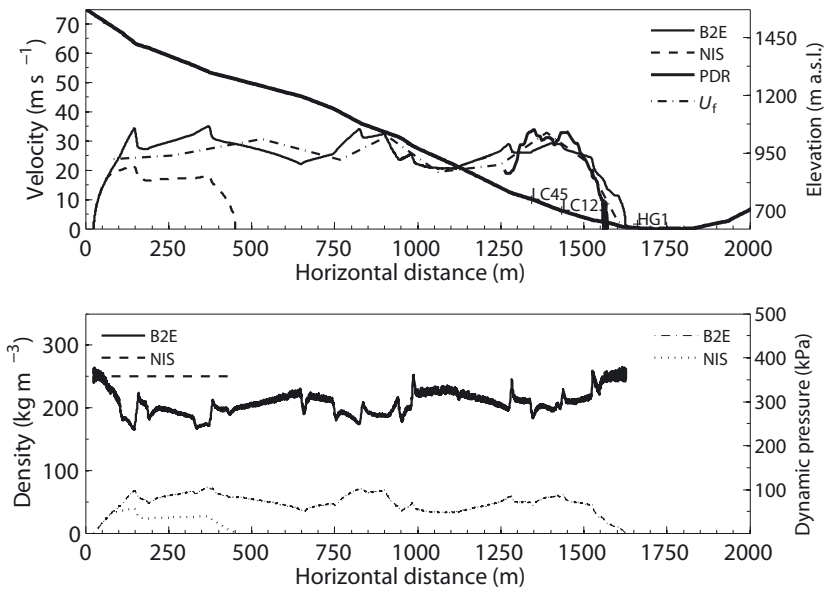

Fig. 5. Measurements and simulations of the 16 April 2005 avalanche event at Ryggfonn, Norway. The front velocity $\left(U_{\mathrm{f}}\right)$ was estimated from video footage. Data from PDR are also available below $800 \mathrm{~m}$ a.s.l. (Gauer and others, 2007a,b). The same model parameters were used in both simulations, but fluidization was disabled in the simulation labelled 'NIS'.

A large, extremely rare avalanche (return period 5001000 years) occurred on 27 January 1994 at Bleie, southern Norway (Lied and others, 1998). Only if large quantities of snow fall at freezing temperatures down to sea level can an avalanche cross the $1 \mathrm{~km}$ long slope inclined at only $10^{\circ}$ and reach the steeper final slope to the fjord (Fig. 7). We found that B2E can simulate this avalanche with the same set of parameters as VdIS and Rüchitobel, except that fluidization needs to be encouraged by specifying a large lift coefficient, i.e. $C_{\mathrm{L}}=2.0$. The simulations may overestimate the pressure at the location of the destroyed houses, but no reliable upper bound could be deduced from the observations.

With the same parameters but without fluidization (NIS model), the runout distance is shortened by more than $2 \mathrm{~km}$ (Fig. 7). With the PCM-type model, parameter combinations that allow the avalanche to reach the fjord had a very low $\mu$ of 0.15 and $M / D=750 \mathrm{~m}$ (Lied and others, 1998). In that case, snow entrainment was not included explicitly. However, it can be considered implicitly included, as the choice of $M / D$ was relatively low in comparison to the used $\mu$ value. We observe a marked difference in velocity on the plateau between the PCM simulation and the B2E simulation presented here. The predicted dynamic pressure in the area around the farm is similar in both simulations, but the runout distance for the PCM model is longer.

\section{DISCUSSION AND CONCLUSIONS}

The simple block model proposed in this paper is limited in many ways. First of all, relevant processes such as granulation (Gubler, 1987) are disregarded. The lateral spreading and longitudinal stretching of the flow, which is strongly enhanced if the head fluidizes, are not captured at all; nor does the model consider how entrained mass is redistributed over the avalanche body. Work on implementing the concepts of B2E in a 2-D depth-averaged code is in progress. Much of this task is conceptually straightforward, but some extensions to the original NIS rheology are required to ensure invariance under coordinate transformations, to 

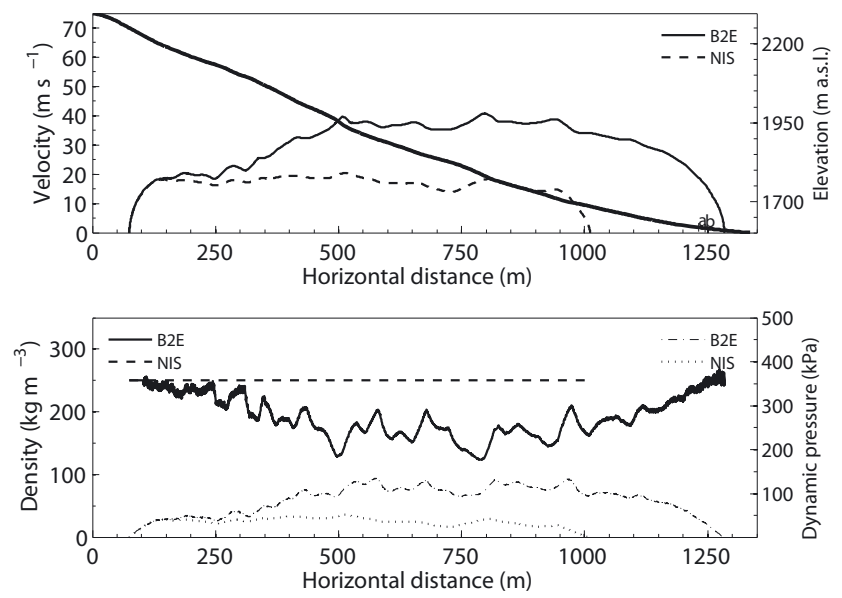

Fig. 6. Simulations of the 18 January 2006 avalanche event in the Rüchitobel, Davos, Switzerland. With the same parameter values as for Ryggfonn and Vallée de la Sionne, except for a lower value of $C_{\mathrm{L}}$, the runout distance is overestimated by $50 \mathrm{~m}$. However, the speed of the fluidized part at $1750 \mathrm{~m}$ a.s.l. is within the estimated range. If fluidization is suppressed (NIS model) with otherwise equal parameters, the estimated speed of the dense part at the same location is well captured.

maintain hyperbolicity even as $h \rightarrow 0$ and to prevent unphysical longitudinal stresses at high shear rates.

In order to put the model on a firm foundation, theoretical and experimental work should be combined. Chute experiments designed to explore the fluidized regime will need rather long chutes with variable inclination, and it would be most interesting if the air density could be varied over a wide range (e.g. by varying the air pressure or replacing the air by a heavy gas in a hermetically sealed chute). Velocity and density profiles need to be measured at various locations. In-depth analysis of measurements from the full-scale test sites will continue to provide valuable insight, especially if densities are measured simultaneously with the velocity profile and impact pressure. Similar measurements in the upper track would provide insight into the onset of fluidization. Complementary information on the topographic and climatic conditions for fluidization can be gained by investigating the deposits of considerable numbers of small to medium-sized avalanches (Issler and others, 2008).

The two most critical uncertainties concern the dependence of the rheological parameters on concentration and the excess pore pressure due to aerodynamic effects. The former can be addressed by extending the discrete-particle simulations of Campbell and Gong (1986) to three dimensions and eventually to dispersed particle sizes. Concerning the aerodynamic forces, simulations of the airflow around and through a porous blunt body will constrain the value of the lift coefficient $C_{\mathrm{L}}$. In addition, a fast compression or stretching of the avalanche body can contribute to the build-up of excess pore pressure. To account for this, an additional equation for the pore pressure may be required.

$\mathrm{B} 2 \mathrm{E}$ is too simplistic to allow a definitive assessment of the consequences that recognition of the fluidized flow regime will have on avalanche hazard mapping, but several preliminary inferences can be made. (1) Avalanche simulations will become more objective than with conventional models because all parameters vary in narrow and physically reasonable ranges. (2) B2E seems to reproduce the
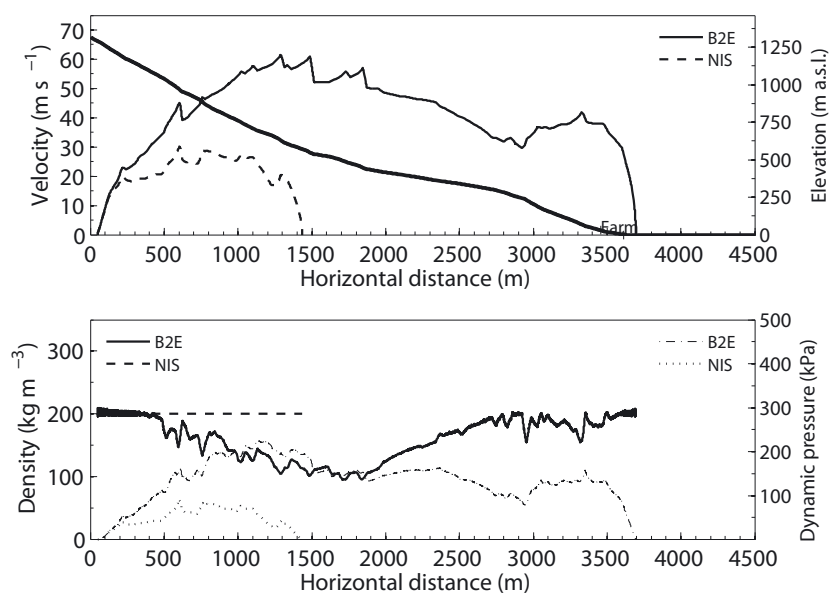

Fig. 7. Simulations of the extreme avalanche at Bleie, Norway, on 27 January 1994. Parameters for B2E are the same as those used at Ryggfonn and Vallée de la Sionne, except for a higher value of $C_{\mathrm{L}}$ needed for sufficient fluidization. The simulation labelled 'NIS' uses the same parameter values, but fluidization is suppressed.

speed quite accurately along the entire path. In conjunction with realistic density predictions from the model, this leads to more reliable pressure distributions. (3) In special topographical situations (e.g. Bleie), the model is able to correctly predict the much longer runout distance of extreme events with physically plausible model parameters. (4) B2E will often predict pressure distributions in the runout zone that differ significantly from those of conventional models. At the present stage of development, B2E predictions may be higher or lower than standard predictions, depending on topography. It will be crucial to obtain reliable density measurements in the fluidized layer to settle this issue.

Avalanche models that correctly capture the essential flow processes may have large predictive power in a wide range of situations. Their parameters, even though numerous, are linked to measurable snow or terrain properties and vary within narrow ranges. Modelling transitions to and from the fluidized regime as well as entrainment appear to be two of the key ingredients in future models. Gubler (1987) discussed a number of further important processes that ought to be included, such as progressive comminution of snow particles. Our findings confirm his conclusion that much of the bewildering complexity of snow avalanche behaviour can be captured by a physically meaningful, reliable and practically usable dynamical model.

\section{ACKNOWLEDGEMENTS}

A substantial part of this work was supported by the European Union through the Framework Programme 5 (FP5) project SATSIE (Avalanche Studies and Model Validation in Europe, EVG1-CT-2002-00059). Additional support was provided by the European Science Foundation (ESF) Integrated Project EUROMARGINS, while the writing was made possible by a special grant from the directorate of the Norwegian Geotechnical Institute (NGI). We thank M. Barbolini, H. Gubler, F. Irgens, M. Kern, A. Moe, M. Naaim, M. Schaer and our colleagues at the NGI Avalanche Group for enjoyable and helpful discussions on this topic. M. Kern provided a careful review of the manuscript. This is ICG publication No. 198 from the International Centre for Geohazards. 


\section{REFERENCES}

Barbolini, M., A. Biancardi, F. Cappabianca, L. Natale and M. Pagliardi. 2005. Laboratory study of erosion processes in snow avalanches. Cold Reg. Sci. Technol., 43(1-2), 1-9.

Campbell, C.S. and A. Gong. 1986. The stress tensor in a twodimensional granular shear flow. J. Fluid Mech. 164, 107-125.

Gauer, P., K. Lied and K. Kristensen. 2007a. On avalanche measurements at the Norwegian full-scale test-site Ryggfonn. Cold Reg. Sci. Technol., 51(2-3), 138-155.

Gauer, P. and 7 others. 2007b. On full-scale avalanche measurements at the Ryggfonn test site, Norway. Cold Reg. Sci. Technol., 49(1), 39-53.

Gauer, P., M. Kern, K. Kristensen, K. Lied, L. Rammer and H. Schreiber. 2007c. On pulsed Doppler radar measurements of avalanches and their implication to avalanche dynamics. Cold Reg. Sci. Technol., 50(1-3), 55-71.

Gubler, H. 1987. Measurements and modelling of snow avalanche speeds. IAHS Publ. 162 (Symposium at Davos 1986 - Avalanche Formation, Movement and Effects), 405-420.

Hampton, M.A. 1972. The role of subaqueous debris flow in generating turbidity currents. J. Sediment. Petrol., 42(4), 775-793.

Issler, D., P. Gauer, M. Schaer and S. Keller. 1996. Staublawinenereignisse im Winter 1995: Seewis (GR), Adelboden (BE) und Col du Pillon (VD). Davos, Eidgenössisches Institut für Schnee- und Lawinenforschung. (Internal Report 694.)

Issler, D., A. Errera, S. Priano, H. Gubler, B. Teufen and B. Krummenacher. 2008. Inferences on flow mechanisms from snow avalanche deposits. Ann. Glaciol., 49. (see paper in this volume).
Lied, K., B. Instanes, U. Domaas and C.B. Harbitz. 1998. Snow avalanche at Bleie, Ullensvang, January 1994. In Hestnes, E., ed. 25 Years of Snow Avalanche Research at NGI. Proceedings of the Anniversary Conference, 12-16 May 1998, Voss, Norway. Oslo, Norwegian Geotechnical Institute, 175-181. (NGI Publication 203.)

Mellor, M. 1977. Engineering properties of snow. J. Glaciol., 19(81), 15-66.

Nishimura, K., S. Keller, J. McElwaine and Y. Nohguchi. 1998. Pingpong ball avalanche at a ski jump. Granular Matter, 1(2), 51-56.

Norem, H., F. Irgens, and B. Schieldrop. 1987. A continuum model for calculating snow avalanche velocities. IAHS Publ. 162 (Symposium at Davos 1986 - Avalanche Formation, Movement and Effects), 363-379.

Pasquarell, G.C., N.L. Ackermann, H.H. Shen and M.A. Hopkins. 1988. Collisional stress in granular flows: Bagnold revisited. ASCE J. Eng. Mech. Div., 114(1), 49-64.

Perla, R., T.T. Cheng and D.M. McClung. 1980. A two-parameter model of snow avalanche motion. J. Glaciol., 26(94), 197-207.

Rajchenbach, J. 2003. Dense, rapid flows of inelastic grains under gravity. Phys. Rev. Lett., 90(14), 144302.

Savage, S.B. and M. Sayed. 1984. Stresses developed by dry cohesionless granular materials sheared in an annular shear cell. J. Fluid Mech. 142, 392-430.

Schaer, M. and D. Issler. 2001. Particle densities, velocities and size distributions in large avalanches from impact-sensor measurements. Ann. Glaciol., 32, 321-327.

Vallet, J., U. Gruber and F. Dufour. 2001. Photogrammetric avalanche volume measurements at Vallée de la Sionne, Switzerland. Ann. Glaciol., 32, 141-146. 